MATHEMATICS OF COMPUTATION

Volume 70, Number 233 , Pages 205-221

S $0025-5718(00) 01226-6$

Article electronically published on March 3, 2000

\title{
NUMERICAL INDEFINITE INTEGRATION OF FUNCTIONS WITH SINGULARITIES
}

\author{
AEYOUNG PARK JANG AND SEYMOUR HABER
}

\begin{abstract}
We derive an indefinite quadrature formula, based on a theorem of Ganelius, for $H^{p}$ functions, for $p>1$, over the interval $(-1,1)$. The main factor in the error of our indefinite quadrature formula is $O\left(e^{-\pi \sqrt{N / q}}\right)$, with $2 N$ nodes and $\frac{1}{p}+\frac{1}{q}=1$. The convergence rate of our formula is better than that of the Stenger-type formulas by a factor of $\sqrt{2}$ in the constant of the exponential. We conjecture that our formula has the best possible value for that constant. The results of numerical examples show that our indefinite quadrature formula is better than Haber's indefinite quadrature formula for $H^{p}$-functions.
\end{abstract}

\section{INTRODUCTION}

For $p>1, H^{p}$ consists of those functions $f$ which are analytic in the unit disc in the complex plane, and for which

$$
\|f\|_{p}=\lim _{r \rightarrow 1^{-}}\left(\frac{1}{2 \pi} \int_{0}^{2 \pi}\left|f\left(r e^{i \theta}\right)\right|^{p} d \theta\right)^{\frac{1}{p}}<\infty .
$$

Thus $H^{p}$ contains functions which may have singularities on the boundary of the unit disc. The $H^{p}$ spaces are well-known spaces of functions in analytic function theory and important in dealing with integrals over $(-1,1)$ of functions that have singularities at endpoints.

Schwartz 14] suggested the "tanh rule" for numerical definite integration over $(-1,1)$. It was studied by Stenger (see [18] and the references therein) for several classes of analytic functions. Haber [8] studied its convergence for $H^{2}$ integrands, for which its convergence rate is $\exp (-\pi \sqrt{N / 2})$. Bojanov [3, 4] studied quadrature formulas for $H^{\infty}$ functions, obtaining formulas with similar convergence rates. Loeb and Werner [11] developed similar formulas for $H^{p}$ functions, deriving them from Newman's seminal paper [12] on rational approximation of $|x|$. Newman [13] also showed that faster convergence than $\exp (-\mathcal{C} \sqrt{N})$ is not possible for the $H^{p}$ classes, and gave upper and lower bounds for the constant $\mathcal{C}$ in the exponent. Sikorski, Stenger and Schwing [15, 16, 17 gave formulas and algorithms for $H^{p}$ classes of several complex domains. For the unit disk, Andersson [1] determined the best possible value of $\mathcal{C}$, for each $p$.

Received by the editor May 19, 1998 and, in revised form, January 4, 1999.

2000 Mathematics Subject Classification. Primary 65D30; Secondary 41A55.

Key words and phrases. Indefinite quadrature formula, $H^{p}$ functions, singularity.

(C)2000 American Mathematical Society 
There is generally another factor, of the form $N^{\mathcal{C}^{\prime}}$, in these error bounds (see, e.g., our theorem below). Andersson and Bojanov [2] determined the best possible values for $\mathcal{C}^{\prime}$.

Stenger's approach - part of a wide-ranging development of new methods in numerical analysis using the Whittaker Cardinal Function - yielded formulas for numerical indefinite integration over $(-1,1)$. Such formulas were given by Stenger 18], Kearfott [10, and Haber [9]. These also converge at $\exp (-\mathcal{C} \sqrt{N})$ rates. But for each $H^{p}$ class, the constant $\mathcal{C}$ for these ("sinc-type") indefinite-quadrature formulas is lower by a factor of $\sqrt{2}$ than the constant for the definite-quadrature formula.

It is also by a factor of $\sqrt{2}$ in the exponent, that Andersson's optimal definitequadrature formulas converge faster than the sinc-type definite-quadrature formulas. And our present indefinite-quadrature formula improves on the sinc-type indefinite-quadrature formulas. Our formula is based on the same theorem of Ganelius 6] that Andersson used (Lemma 1, below) and uses other ideas of Andersson's. So we conjecture that our formula is optimal, as far as the coefficient $\mathcal{C}$ in the exponent is concerned.

More precisely, we conjecture that for any $p$ in $(1, \infty)$ and any increasing infinite sequence of positive integers $M_{1}, M_{2}, \ldots$, and sequence of indefinite quadrature formulas

$$
Q_{i}(f, t)=\sum_{k=1}^{M_{i}} a_{i, k}(t) f\left(b_{i, k}\right),
$$

the error

$$
\sup _{|t| \leq 1}\left|\int_{-1}^{t} f(x) d x-Q_{i}(f, t)\right|
$$

is greater than

$$
\mathcal{K}(f) \exp \left(-\mathcal{C} \sqrt{M_{i}}\right) \text { for all sufficiently large } i
$$

if $\mathcal{C}$ is greater than $\pi / \sqrt{2 q}$. (The last being the constant given by our formula, noting that $M$ would be $2 N$.)

In Section 2 we derive the indefinite quadrature formula and its error bound for $H^{p}$ functions. In Section 3 we explain the nodes of our formula and compare the convergence rates of the indefinite quadrature formula derived in this paper with those of Haber's indefinite quadrature formula. In the Appendix we prove a modified form of Ganelius's theorem which also corrects a small error in Ganelius's discussion in [6].

\section{Derivation of the indefinite quadrature Formula}

For $f \in H^{p}, p>1$, we start by defining the integral

$$
I(x)=\frac{1}{2 \pi i} \int_{|z|=1} \frac{1}{B_{n}(z)} \cdot \frac{f(z)}{z-x} d z \quad \text { for }-1<x<1,
$$

using the fact that functions in $H^{p}$ have limiting values almost everywhere on $|z|=1$. See Duren [5] Theorems 1.3, 2.2 and 3.6]. Here

$$
B_{n}(z)=\prod_{-N}^{N} \frac{z-b_{k}}{1-b_{k} z}, \quad \text { with } n=2 N,
$$


and

$$
b_{k}=\sqrt{\frac{1-a_{k}}{1+a_{k}}}, \quad b_{-k}=-b_{k},
$$

and $a_{k}$ are those defined in Lemma 1 below, using $r=\left(1-(2 N)^{(-1 / 2)}\right) / q$. Here and below the prime on a product (or sum) indicates that the index value $k=0$ is excluded.

We evaluate the integral by residues. The singularities inside the unit disc are at $z=x$ with residue $f(x) / B_{n}(x)$ and at $z=b_{k}, k$ any nonzero integer between $-N$ and $N$, with residue

$$
\rho_{k} \cdot \frac{f\left(b_{k}\right)}{b_{k}-x}
$$

where

$$
\rho_{k}=\prod_{l=-N}^{N}\left(1-b_{l} b_{k}\right) / \prod_{\substack{l=-N \\ l \neq k}}^{N}\left(b_{k}-b_{l}\right) .
$$

After some algebra we get the equation

$$
f(x)=\sum_{k=-N}^{N} \rho_{k} \cdot \frac{B_{n}(x)}{\left(x-b_{k}\right)} \cdot f\left(b_{k}\right)+B_{n}(x) I(x) .
$$

Now we know that

$$
\frac{B_{n}(x)}{x-b_{k}}=\sum_{m=-N}^{N} \rho_{m} \cdot \frac{1}{1-b_{k} b_{m}} \cdot \frac{1}{1-b_{m} x},
$$

so integrating from -1 to $t$ in (1) gives us

$$
\begin{gathered}
\int_{-1}^{t} f(x) d x=\sum_{k=-N}^{N} f\left(b_{k}\right) \cdot\left[\rho_{k} \sum_{m=-N}^{N} \frac{\rho_{m}}{b_{m}\left(1-b_{k} b_{m}\right)} \cdot \log \frac{1+b_{m}}{1-b_{m} t}\right]+R(t), \\
\text { where } R(t)=\frac{1}{2 \pi i} \int_{-1}^{t} \int_{|z|=1} \frac{f(z) B_{n}(x)}{(z-x) B_{n}(z)} d z d x .
\end{gathered}
$$

Theorem. If $f$ is in $H^{p}$ with $1<p<\infty$ and $q=p /(p-1)$, then

$$
\begin{gathered}
\int_{-1}^{t} f(x) d x=\sum_{k=-N}^{N} f\left(b_{k}\right) \cdot\left[\rho_{k} \sum_{m=-N}^{N} \frac{\rho_{m}}{b_{m}\left(1-b_{k} b_{m}\right)} \cdot \log \frac{1+b_{m}}{1-b_{m} t}\right] \\
+O\left(N^{\frac{1}{2 q}} \exp (-\pi \sqrt{N / q})\right),
\end{gathered}
$$

uniformly in $[-1,1]$.

We shall prove this with the aid of the following lemmas.

Lemma 1 (Ganelius). For $0<r<1$ and $N$ a positive integer, the numbers $a_{k}$ that are defined by
a. $N_{o}=N-\left\lceil\frac{\pi}{4} \sqrt{N r}\right\rceil$,
b. $\varphi$ is the function defined by $\varphi(x)=\exp (\pi \sqrt{x / r})$, and 
$c$.

$$
a_{k}= \begin{cases}\varphi(k-1) / \varphi\left(N_{o}\right), & \text { for } k=1,2,3, \ldots, N_{o} \\ \varphi(k-3 / 2) / \varphi\left(N_{o}\right), & \text { for } k=N_{o}+1, \\ 1-\frac{k-N_{o}-1}{5\left(N-N_{o}-1\right)}, & \text { for } N_{o}+2 \leq k \leq N\end{cases}
$$

satisfy the inequality

$$
\max _{x \in[0,1]} x^{r} \prod_{1}^{N}\left|\frac{x-a_{k}}{x+a_{k}}\right| \leq \mathcal{C} \exp (-\pi \sqrt{N r}),
$$

where $\mathcal{C}$ depends on $r$ but not on $N$.

We prove this modified form of Ganelius's theorem in the Appendix. Transforming this result to the unit disk (following Loeb and Werner [11]) by

$$
x=\frac{1-z^{2}}{1+z^{2}},
$$

we find that

$$
\max _{z \in[-1,1]}\left(1-z^{2}\right)^{r} \prod_{-N}^{N}\left|\frac{z-b_{k}}{1-b_{k} z}\right| \leq \mathcal{C} \exp (-\pi \sqrt{N r}) .
$$

Lemma 2. For any positive number $q$,

$$
\int_{-\pi}^{\pi}\left(\int_{-1}^{1} \frac{1}{\left|e^{i \theta}-x\right|} d x\right)^{q} d \theta<\infty
$$

Proof.

$$
\begin{aligned}
\int_{-1}^{1} \frac{1}{\left|e^{i \theta}-x\right|} d x & =\int_{-1}^{1} \frac{1}{\sqrt{1-2 x \cos \theta+x^{2}}} d x \\
& =\log \frac{\sqrt{2-2 \cos \theta}+1-\cos \theta}{\sqrt{2+2 \cos \theta}-1-\cos \theta} .
\end{aligned}
$$

The last expression becomes infinite when $\theta$ approaches $0, \pi$, or $-\pi$. But it is like $\log \frac{1}{|\theta|}$ as $\theta \rightarrow 0, \log \frac{1}{|\pi-\theta|}$ as $\theta \rightarrow \pi$, and $\log \frac{1}{|\pi+\theta|}$ as $\theta \rightarrow-\pi$. Thus the singularities are only logarithmic in size, and it follows that

$$
\int_{-\pi}^{\pi}\left(\log \frac{\sqrt{2-2 \cos \theta}+1-\cos \theta}{\sqrt{2+2 \cos \theta}-1-\cos \theta}\right)^{q} d \theta<\infty, \quad \text { for every positive } q .
$$

Lemma 3. For $q>1$

$$
I(r, q)=\int_{-\pi}^{\pi}\left(\int_{-1}^{1} \frac{d x}{\left(1-x^{2}\right)^{r} \sqrt{1-2 x \cos \theta+x^{2}}}\right)^{q} d \theta<\frac{\mathcal{C}}{1-q r},
$$

for $\frac{1}{10 q}<r<\frac{1}{q} ; \mathcal{C}$ depends on $q$ alone.

Proof. Since the integrand is even in $\theta$, we can limit our attention to the upper half of the integration region. Introducing the numbers $\epsilon$ and $\delta$ in $(0,1)$ and setting

$$
h(x, \theta)=\frac{1}{\left(1-x^{2}\right)^{r} \sqrt{1-2 x \cos \theta+x^{2}}},
$$


we write

$$
\begin{aligned}
\frac{1}{2} I(r, q)= & \int_{0}^{\pi}\left(\int_{-1}^{1} \frac{d x}{\left(1-x^{2}\right)^{r} \sqrt{1-2 x \cos \theta+x^{2}}}\right)^{q} d \theta \\
= & \int_{0}^{\epsilon}\left(\int_{-1}^{1} h(x, \theta) d x\right)^{q} d \theta+\int_{\epsilon}^{\pi-\epsilon}\left(\int_{-1}^{1} h(x, \theta) d x\right)^{q} d \theta \\
& +\int_{\pi-\epsilon}^{\pi}\left(\int_{-1}^{1} h(x, \theta) d x\right)^{q} d \theta
\end{aligned}
$$

Then noting that $h(-x, \pi-\theta)=h(x, \theta)$, we write

$$
\begin{aligned}
\frac{1}{2} I(r, q)= & \int_{0}^{\epsilon}\left(\int_{-1}^{1-\delta} h(x, \theta) d x+\int_{1-\delta}^{1} h(x, \theta) d x\right)^{q} d \theta \\
& +2 \int_{\epsilon}^{\pi-\epsilon}\left(\int_{-1}^{1} h(x, \theta) d x\right)^{q} d \theta .
\end{aligned}
$$

In the first integral of $(2)$, noting that $x \leq 1-\delta$ and so $\sqrt{1-2 x \cos \theta+x^{2}} \geq \delta$, we have

$$
\int_{-1}^{1-\delta} h(x, \theta) d x \leq \int_{-1}^{1-\delta} \frac{d x}{\delta\left(1-x^{2}\right)^{r}} \leq \frac{2}{\delta(1-r)}
$$

Letting $x=1-y$ in the first integral of (2), we write

$$
\int_{1-\delta}^{1} h(x, \theta) d x=\int_{0}^{\delta} \frac{d y}{(2-y)^{r} y^{r} \sqrt{y^{2}+2(1-y)(1-\cos \theta)}} .
$$

Observing that $y^{2}+2(1-y)(1-\cos \theta) \geq y^{2}+2(1-\delta)(1-\cos \theta) \geq y^{2}+\left(\sqrt{\frac{2(1-\delta)}{3}}\right)^{2} \theta^{2}$, and letting $B=\sqrt{\frac{2(1-\delta)}{3}}$ and $t=\frac{y}{B \theta}$, we get

$$
\begin{aligned}
\int_{1-\delta}^{1} h(x, \theta) d x & \leq \int_{0}^{\delta} \frac{d y}{y^{r} \sqrt{y^{2}+B^{2} \theta^{2}}} \\
& \leq \frac{1}{B^{r} \theta^{r}} \int_{0}^{\frac{\delta}{B \theta}} \frac{d t}{t^{r} \sqrt{t^{2}+1}} \\
& \leq \frac{1}{B^{r} \theta^{r}} \int_{0}^{\infty} \frac{d t}{t^{r} \sqrt{t^{2}+1}} \\
& \leq \frac{1}{(B \theta)^{r}} \cdot\left(\frac{1}{r}+\frac{1}{1-r}\right) .
\end{aligned}
$$

So in the first integral of (2), we get

$$
\begin{aligned}
\int_{0}^{\epsilon}\left(\int_{-1}^{1} h(x, \theta) d x\right)^{q} d \theta & \leq \int_{0}^{\epsilon}\left[\frac{2}{\delta(1-r)}+\frac{1}{(B \theta)^{r}} \cdot\left(\frac{1}{r}+\frac{1}{1-r}\right)\right]^{q} d \theta \\
& \leq \int_{0}^{\epsilon}\left[\frac{2}{\delta(1-r)}+\frac{1}{B^{r}} \cdot\left(\frac{1}{r}+\frac{1}{1-r}\right)\right]^{q} \cdot\left(\frac{1}{\theta^{r}}\right)^{q} d \theta \\
& \leq\left[\frac{2}{\delta(1-r)}+\left(\frac{3}{2(1-\delta)}\right)^{\frac{r}{2}} \cdot\left(\frac{1}{r}+\frac{1}{1-r}\right)\right]^{q} \cdot \frac{1}{1-r q}
\end{aligned}
$$


In the last integral of (2), observing that $\sqrt{1-2 x \cos \theta+x^{2}} \geq \sin \theta \geq \frac{2}{\pi} \epsilon$ for $x \in[-1,1]$ and $\theta \in[\epsilon, \pi-\epsilon]$, we see that

$$
\begin{aligned}
\int_{\epsilon}^{\pi-\epsilon}\left(\int_{-1}^{1} h(x, \theta) d x\right)^{q} d \theta & \leq \int_{\epsilon}^{\pi-\epsilon}\left(\frac{\pi}{2 \epsilon} \int_{-1}^{1} \frac{d x}{\left(1-x^{2}\right)^{r}}\right)^{q} d \theta \\
& \leq \int_{\epsilon}^{\pi-\epsilon}\left(\frac{\pi}{\epsilon(1-r)}\right)^{q} d \theta \leq \pi\left(\frac{\pi}{\epsilon(1-r)}\right)^{q}
\end{aligned}
$$

Combining the two estimates, we see that

$$
I(r, q) \leq \mathcal{C} \cdot\left(\frac{1}{r}+\frac{1}{1-r}\right)^{q} \cdot \frac{1}{1-r q}
$$

for some $\mathcal{C}(q)$ independent of $r$. With $r$ between $1 /(10 q)$ and $1 / q$, the quantity $1 / r+1 /(1-r)$ is further bounded by $10 q+q /(q-1)$. The proof of the lemma is complete.

Proof of theorem. The error term of the indefinite quadrature formula is

$$
|R(t)|=\left|\frac{1}{2 \pi i} \int_{-1}^{t} \int_{|z|=1} \frac{f(z) B_{n}(x)}{(z-x) B_{n}(z)} d z d x\right|
$$

For any $p>1$, we write

$$
\begin{aligned}
\int_{|z|=1} \int_{-1}^{1}\left|\frac{f(z) B_{n}(x)}{(z-x) B_{n}(z)}\right| d x|d z| & =\int_{|z|=1} \int_{-1}^{1}\left|\frac{f(z) B_{n}(x)}{(z-x)}\right| d x|d z| \\
& =\int_{-\pi}^{\pi} \int_{-1}^{1}\left|\frac{B_{n}(x) f\left(e^{i \theta}\right)}{e^{i \theta}-x}\right| d x d \theta \\
& \leq\left(\int_{-\pi}^{\pi}\left|f\left(e^{i \theta}\right)\right|^{p} d \theta\right)^{\frac{1}{p}}\left(\int_{-\pi}^{\pi}\left(\int_{-1}^{1} \frac{d x}{\left|e^{i \theta}-x\right|}\right)^{q} d \theta\right)^{\frac{1}{q}} \\
& \leq \mathcal{C}\left(\int_{-\pi}^{\pi}\left(\int_{-1}^{1} \frac{d x}{\left|e^{i \theta}-x\right|}\right)^{q} d \theta\right)^{\frac{1}{q}} .
\end{aligned}
$$

Applying Lemma 2,

$$
\int_{|z|=1} \int_{-1}^{t}\left|\frac{f(z) B_{n}(x)}{(z-x) B_{n}(z)}\right| d x|d z|<\infty .
$$


Therefore, by Fubini's theorem we can exchange the order of integration in $R(t)$ for $p>1$. We have

$$
\begin{aligned}
|R(t)| & =\left|\frac{1}{2 \pi i} \int_{|z|=1}\left(\int_{-1}^{t} \frac{B_{n}(x)}{(z-x)} \cdot \frac{f(z)}{B_{n}(z)} d x\right) d z\right| \\
& \leq \frac{1}{2 \pi}\left(\int_{|z|=1}\left|\frac{f(z)}{B_{n}(z)}\right|^{p}|d z|\right)^{\frac{1}{p}} \cdot\left(\int_{|z|=1}\left|\int_{-1}^{t} \frac{B_{n}(x)}{(z-x)} d x\right|^{q}|d z|\right)^{\frac{1}{q}} \\
& \leq \frac{1}{2 \pi}\left(\int_{|z|=1}|f(z)|^{p}|d z|\right)^{\frac{1}{p}} \cdot\left(\int_{|z|=1}\left|\int_{-1}^{t} \frac{B_{n}(x)}{(z-x)} d x\right|^{q}|d z|\right)^{\frac{1}{q}} \\
& \leq \mathcal{C} \cdot\|f\|_{p}\left(\int_{|z|=1}\left|\int_{-1}^{t}\left(1-x^{2}\right)^{r} B_{n}(x) \cdot \frac{d x}{(z-x)\left(1-x^{2}\right)^{r}}\right|^{q}|d z|\right)^{\frac{1}{q}} \\
& \leq \mathcal{C} \cdot\|f\|_{p} \max _{x \in[-1,1]}\left(1-x^{2}\right)^{r} B_{n}(x) \cdot\left(\int_{|z|=1}\left(\int_{-1}^{t} \frac{d x}{|z-x|\left(1-x^{2}\right)^{r}}\right)^{q}|d z|\right)^{\frac{1}{q}} .
\end{aligned}
$$

Using Lemma 1 (Ganelius) and Lemma 3 with $r=\left(1-(2 N)^{(-1 / 2)}\right) / q$, we obtain

$$
|R(t)| \leq \mathcal{C} n^{\frac{1}{2 q}} \exp \left(-\pi \sqrt{\frac{n}{2 q}}\right) .
$$

That is, we conclude

$$
|R(t)| \leq \mathcal{C} N^{\frac{1}{2 q}} \exp \left(-\pi \sqrt{\frac{N}{q}}\right)
$$

For $f \in H^{\infty}$ the above reasoning requires one modification: the upper bound $\mathcal{C} /(1-q r)$ in Lemma 3 is replaced by $\mathcal{C} /(1-r)^{2}$. The result is a statement just like the theorem except that the factor $N^{1 / 2 q}$ in the error bound is replaced by $N^{1 / q}=N$.

\section{ThE NODES OF THE INDEFINITE QUADRATURE FORMULA AND COMPUTATIONAL EXAMPLES}

The theorem in Section 2 gives us the indefinite quadrature formula with $2 N$ nodes:

$$
\int_{-1}^{t} f(x) d x \approx \sum_{k=-N}^{N} f\left(b_{k}\right) \cdot\left[\rho_{k} \sum_{m=-N}^{N} \rho_{m} \cdot \frac{1}{b_{m}\left(1-b_{k} b_{m}\right)} \cdot \log \frac{1+b_{m}}{1-b_{m} t}\right],
$$

with $\rho_{k}=\prod_{l=-N}^{N}\left(1-b_{l} b_{k}\right) / \prod_{\substack{l=-N \\ l \neq k}}^{N}\left(b_{k}-b_{l}\right), r=\left(1-(2 N)^{(-1 / 2)}\right) / q$ and $q=\frac{p}{p-1}$.

We observe the following in the proof of Ganelius's lemma. Choosing $N_{o}$ as

$$
N_{o}=N-\left\lceil\frac{\pi}{4} \sqrt{N r}\right\rceil,
$$


the nodes of our indefinite quadrature formula are

$$
\begin{aligned}
& b_{k}=\sqrt{\frac{1-a_{k}}{1+a_{k}}}, \text { for } k=1 \text { to } N \\
& b_{k}=-b_{-k}, \quad \text { for } k=-1 \text { to }-N
\end{aligned}
$$

with $\varphi(x)=\exp (\pi \sqrt{x / r})$ and $a_{k}= \begin{cases}\varphi(k-1) / \varphi\left(N_{o}\right), & \text { for } k=1,2,3, \ldots, N_{o}, \\ \varphi(k-3 / 2) / \varphi\left(N_{o}\right), & \text { for } k=N_{o}+1, \\ 1-\frac{k-N_{o}-1}{5\left(N-N_{o}-1\right)}, & \text { for } N_{o}+2 \leq k \leq N .\end{cases}$

Now, we shall look at the results of applying our indefinite quadrature formula to the following four integrands and compare these with the results of applying Haber's indefinite quadrature formula (A) from [9] to the same integrands, namely

$$
\begin{array}{ll}
f_{1}(x)=\frac{1}{\pi \sqrt{1-x^{2}}}, & f_{2}(x)=\frac{1}{4 \log 2} \log \left(\frac{1+x}{1-x}\right), \\
f_{3}(x)=\frac{\sqrt{1+x^{2}}}{\sqrt{2}+\log (1+\sqrt{2})}, & f_{4}(x)=\frac{2 x}{\pi \sqrt{1-x^{4}}} .
\end{array}
$$

All the functions have singularities at $x= \pm 1$ or $x= \pm i . f_{1}$ is "almost in $H^{2}$ " (in the sense that if its singularities on the unit circle are weakened slightly - for example, by multiplying $f_{1}$ by $\left(1-x^{2}\right)^{\epsilon}$ with $\epsilon$ any positive number - the resulting function is in $H^{2}$ ). Similarly, $f_{2}$ is "almost in $H^{\infty}$ ", $f_{3}$ is in $H^{\infty}$, and $f_{4}$ is "almost in $H^{2}$ ". So we use $q=2$ for $f_{1}$ and $f_{4}$, and $q=1$ for $f_{2}$ and $f_{3}$ to set the value of $r$ in applying our indefinite quadrature formula.

In order to make the convergence rate evident, we apply our indefinite quadrature formula for $N=4,9,16,25, \ldots$. Our error bound suggests that the "convergence ratio" - the ratio of the maximal absolute error for $-1 \leq t \leq 1$ for a given $N$ to that for the next higher $N$-might then be $\exp \left(\frac{\pi}{\sqrt{q}}\right)$ for $p>1$ and $q=\frac{p}{p-1}$.

In each table there is a "maxerr" that presents the maximum of the absolute value of the error of applying the indefinite quadrature formula for each $N$. These were determined as follows. By drawing a graph of the error curve using 201 equally spaced $t$ values over the interval $[-1,1]$, we determined a small interval about the value of $t$ for which the error is greatest in absolute value. We then draw a graph of the error curve over that small interval to locate the maximum point more exactly. Then we iterate this step until we are satisfied that we have the actual "max err" to 3 decimal places at least. The "ratio" in the tables shows the ratio of max err for the previous value of $N$ to max err for the current value. The "max err1" and "ratio1" present the results of applying our indefinite quadrature formula, and the "maxerr2" and "ratio2" present the results of applying Haber's indefinite quadrature formula (A) from 9 .

For these four functions the convergence ratios suggested by our error bound are $\exp (\pi / \sqrt{2}), \exp (\pi), \exp (\pi)$ and $\exp (\pi / \sqrt{2})(\approx 9.2,23.1,23.1$ and 9.2$)$, respectively. The convergence ratios suggested by the upper bound for Haber's formula (A) from [9] are $\exp (\pi / \sqrt{2}), \exp (\pi), \exp (\pi / \sqrt{2})$ and $\exp (\pi / 2)(\approx 9.2,23.1,9.2$ and 4.8 ), respectively (those for $f_{1}$ and $f_{2}$ are so high because the bound in Haber [9] makes use of the analyticity of the integrands in domains extending beyond the unit disk). The computational results are consistent with these suggested ratios for the higher values $N$, which suggests that the error bounds here and in Haber [9] are sharp, giving the actual convergence behaviors of the two formulas for the 
"worst" functions in the specified function spaces. The faster convergence of the new formula is evident in Tables 3 and 4.

Figures 1 and 2 show the error curve - the true indefinite integral minus the approximation - for the integrands $f_{1}$ and $f_{2}$, respectively, using 201 equally spaced $t$ values over the interval $[-1,1]$ for our formula with $N=25$. In the case of the integrand $f_{1}$, we have the maximum of the absolute value of the error of applying our indefinite quadrature formula at $t=1.0$. The maximum of error is $9.50 E-6$, which is out of range in Figure 1. We draw the error curve only for $y \in[4.7512 E-$ $6,4.7528 E-6]$, in order to show movements in the errors more clearly. Figure 2 suggests that the maximum error is near $t=0.96$. But this is misleading. The maximum absolute error for $f_{2}$ is $9.87 E-8$, not $8.94 E-8$ as appears from Figure 2 ; and the maximum occurs at $t=0.9999947$, not at 0.96 . Figures $2-1$ through $2-4$ show how we determined that. Figure 2-1 shows the error curve over the interval $[0.95,1]$. It shows clearly that there is considerable oscillation beyond $t=0.96$, and makes it clear that the maximum occurs somewhere past $t=0.995$. A plot over $[0.995,1]$ (not shown) confirmed that and showed that one must look beyond $t=0.9999$. Figure $2-2$, over the interval $[0.9999,1]$ shows that the maximum occurs for $t>0.99999$. Figure 2-3 over $[0.99999,1]$ confirms that the best local maximum seen is just a bit less than $10^{-7}$ and it occurs at about $t=0.999995$. There is still some worry about possible oscillations nearer to $t=1$, but a plot over $[0.999999,1]$ (not shown), and Figure 2-4 show that the last oscillation is a small one (and incidentally that the maximum is definitely not at $t=1$ ). Similar study of all the error curves yields the numbers in Tables 1 through 4 .

The computations were done on an IBM-type PC in high precision, using UBASIC 8.74.

We wish to thank Professor Yuji Kida and his associates for making the very useful language UBASIC freely available to the mathematical community. 


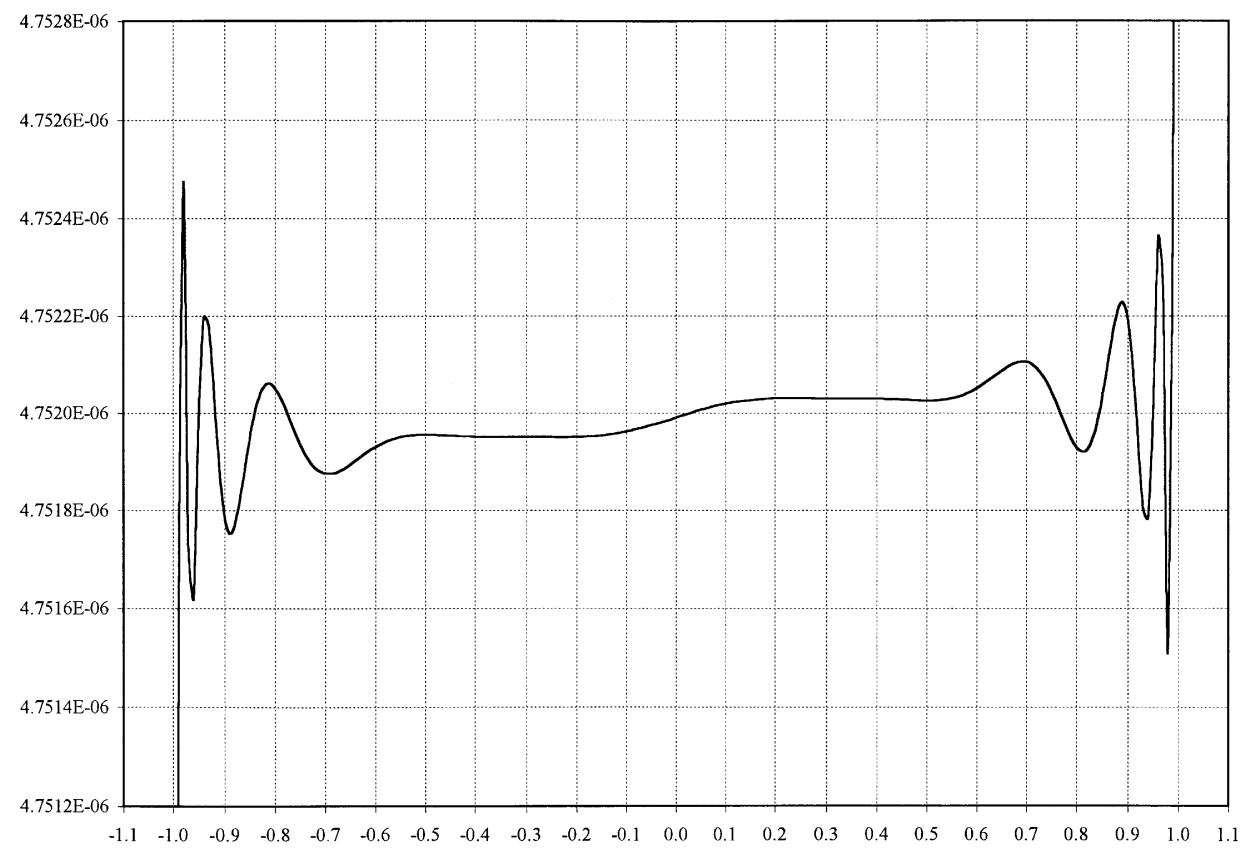

FIGURE 1.

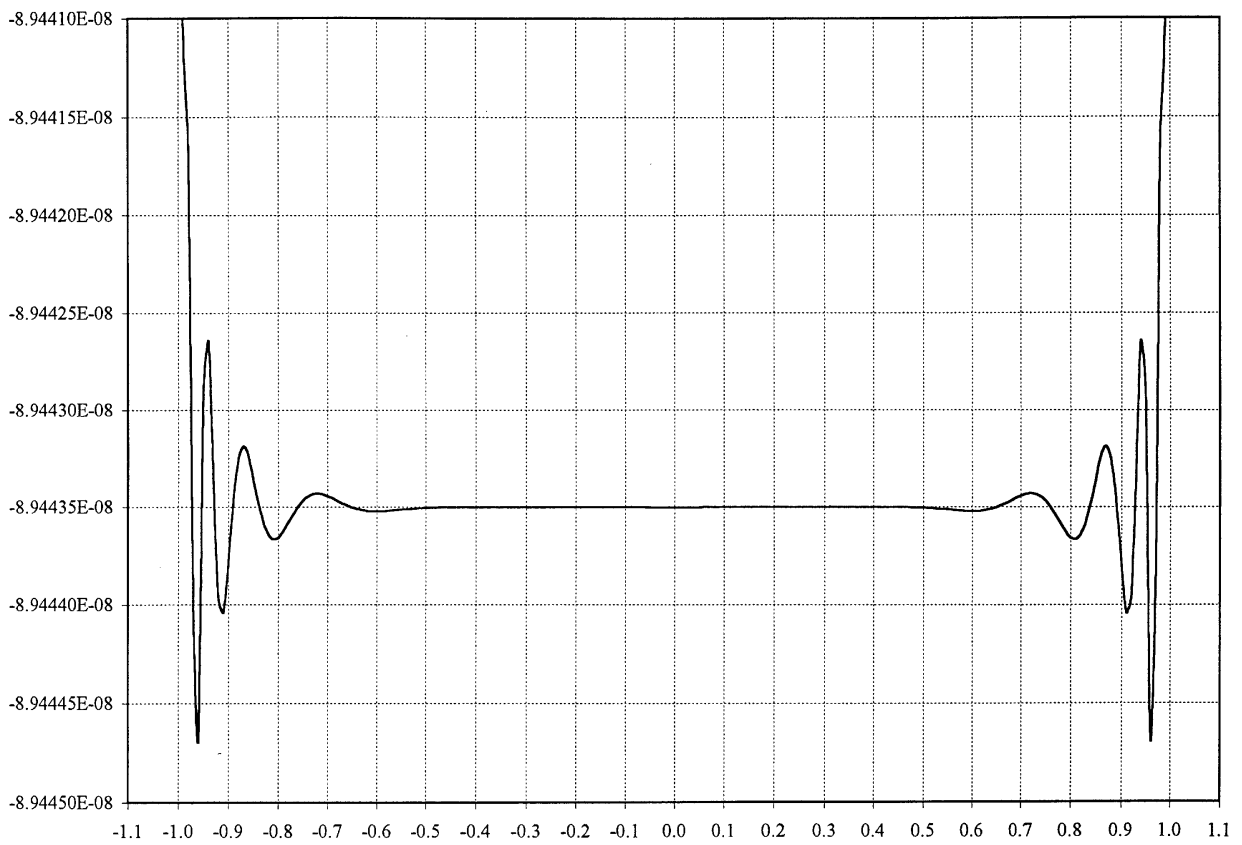

FIGURE 2 . 


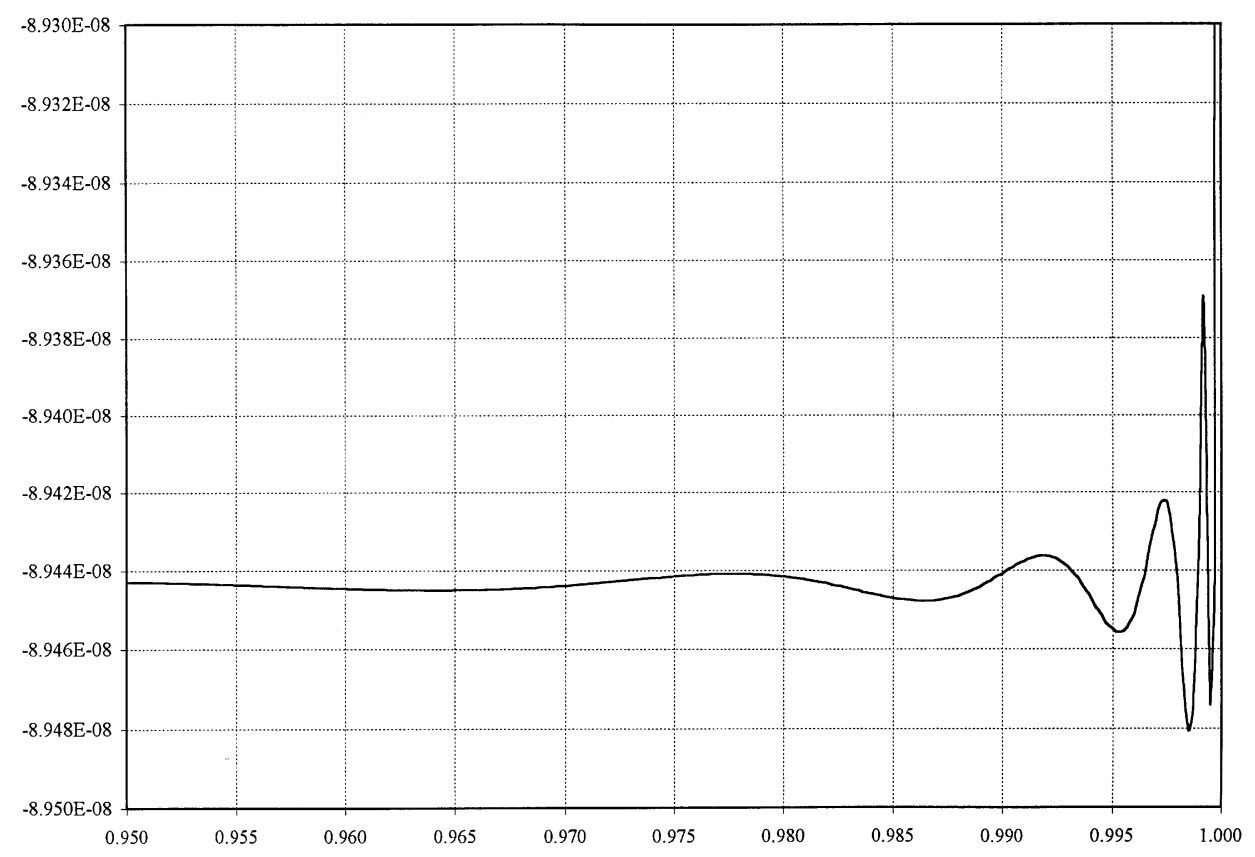

Figure 2-1.

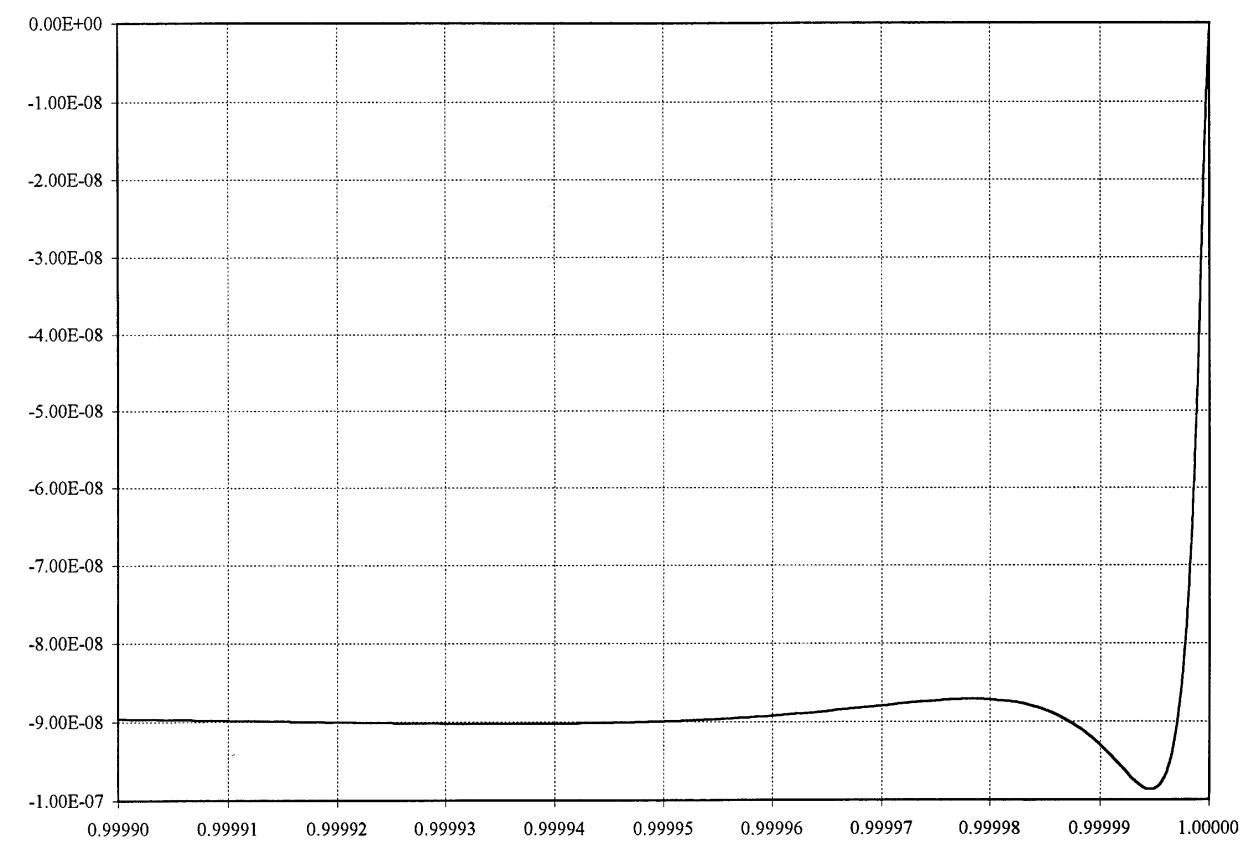

FIGURE 2-2. 


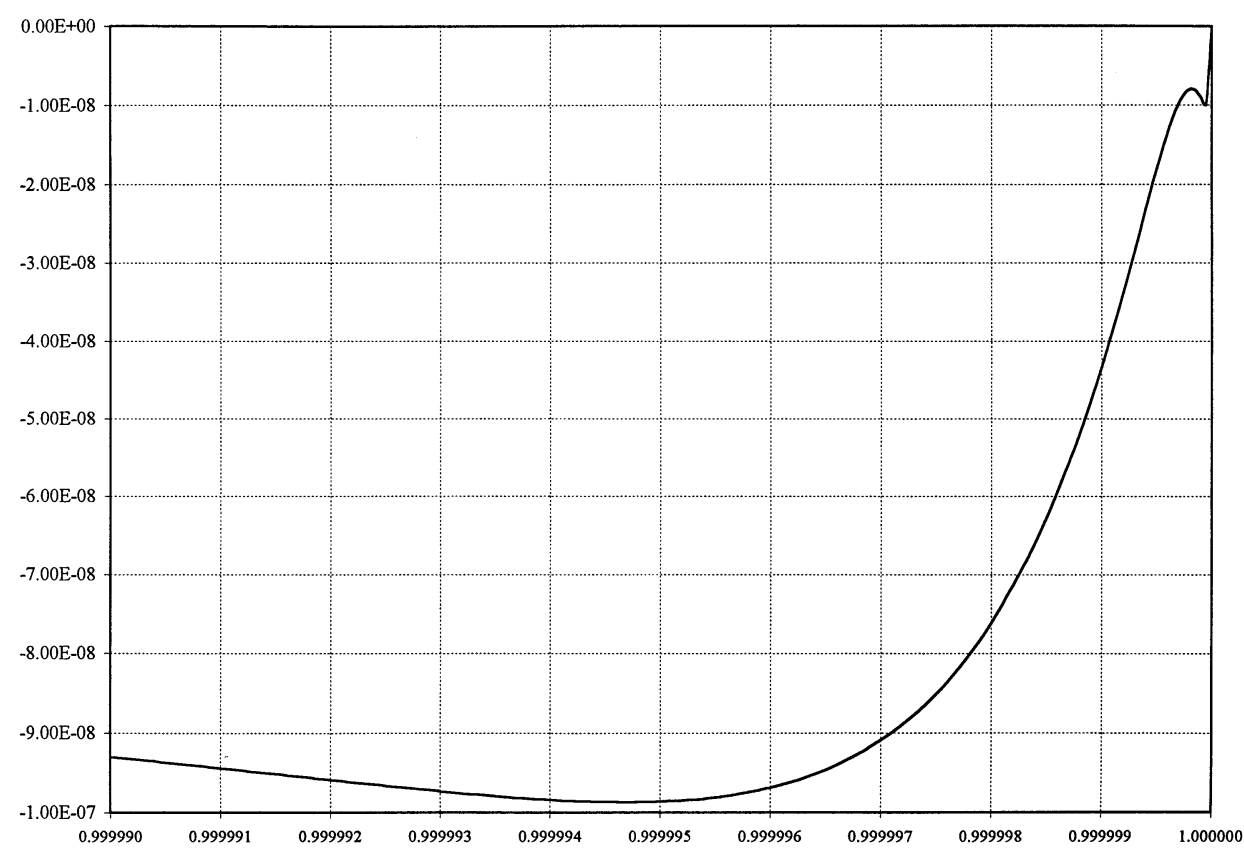

FiguRE 2-3.

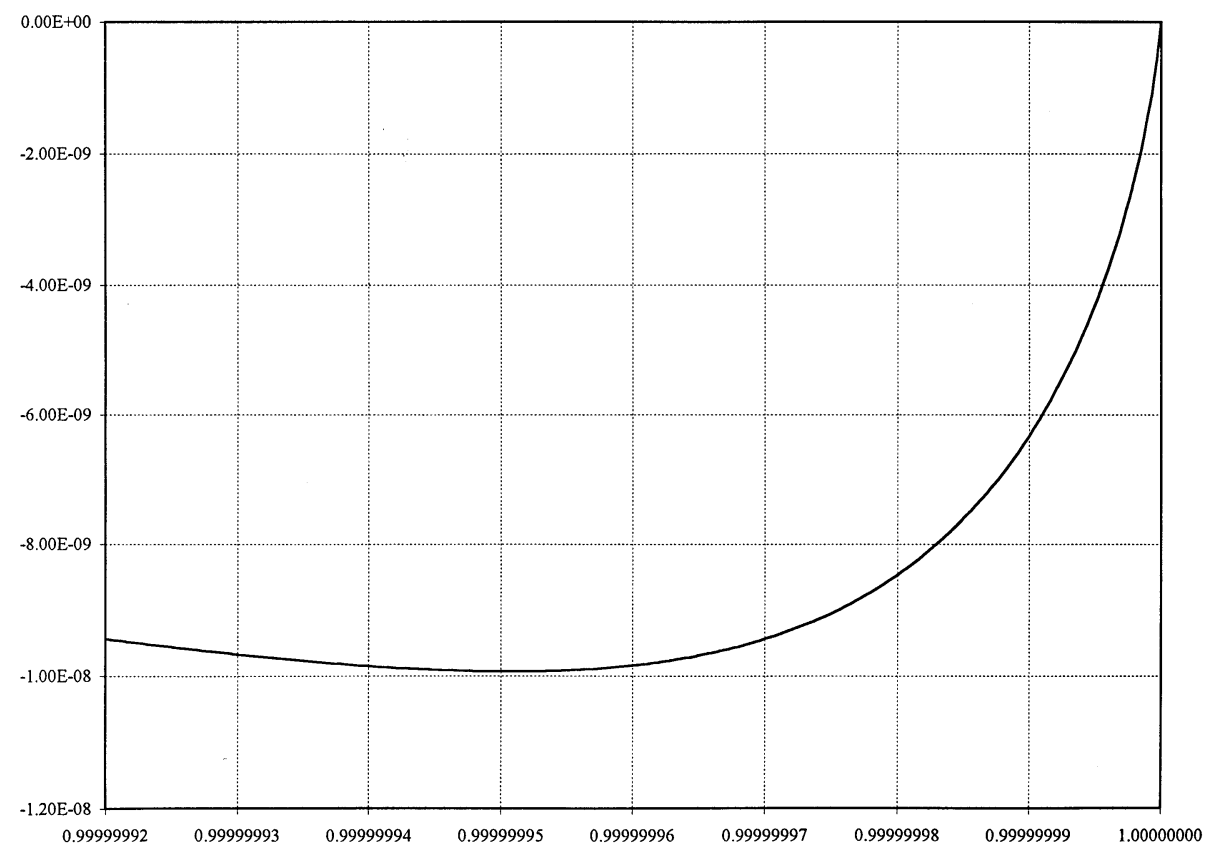

FIGURE 2-4. 
TABLE 1. $f_{1}(x)=\left(\pi \sqrt{1-x^{2}}\right)^{-1}$

\begin{tabular}{rlrcc}
\hline $\mathrm{N}$ & max err1 & ratio1 & max err2 & ratio2 \\
\hline 4 & $1.52 \mathrm{E}-2$ & & $5.80 \mathrm{E}-3$ & \\
9 & $1.14 \mathrm{E}-3$ & 13.3 & $6.67 \mathrm{E}-4$ & 8.7 \\
16 & $1.17 \mathrm{E}-4$ & 9.7 & $7.58 \mathrm{E}-5$ & 8.8 \\
25 & $9.50 \mathrm{E}-6$ & 12.3 & $8.45 \mathrm{E}-6$ & 9.0 \\
36 & $1.06 \mathrm{E}-6$ & 9.0 & $9.34 \mathrm{E}-7$ & 9.0 \\
49 & $9.85 \mathrm{E}-8$ & 10.8 & $1.03 \mathrm{E}-7$ & 9.1 \\
64 & $1.11 \mathrm{E}-8$ & 8.9 & $1.13 \mathrm{E}-8$ & 9.1 \\
81 & $1.08 \mathrm{E}-9$ & 10.3 & & \\
100 & $1.22 \mathrm{E}-10$ & 8.9 & & \\
\hline
\end{tabular}

TABLE 2. $f_{2}(x)=\frac{1}{4 \log 2} \log \left(\frac{1+x}{1-x}\right)$

\begin{tabular}{rllll}
\hline $\mathrm{N}$ & max err1 & ratio1 & max err2 & ratio2 \\
\hline 4 & $4.59 \mathrm{E}-3$ & & $1.06 \mathrm{E}-3$ & \\
9 & $1.25 \mathrm{E}-4$ & 36.7 & $6.01 \mathrm{E}-4$ & 17.6 \\
16 & $2.43 \mathrm{E}-6$ & 51.4 & $3.35 \mathrm{E}-5$ & 17.9 \\
25 & $9.87 \mathrm{E}-8$ & 24.6 & $1.77 \mathrm{E}-6$ & 19.0 \\
36 & $4.09 \mathrm{E}-9$ & 24.1 & $9.10 \mathrm{E}-8$ & 19.4 \\
49 & $1.72 \mathrm{E}-10$ & 23.8 & $4.55 \mathrm{E}-9$ & 20.0 \\
64 & $5.85 \mathrm{E}-12$ & 29.4 & $2.24 \mathrm{E}-10$ & 20.3 \\
81 & $2.55 \mathrm{E}-13$ & 22.9 & $1.08 \mathrm{E}-11$ & 20.6 \\
100 & $1.02 \mathrm{E}-14$ & 25.0 & $5.20 \mathrm{E}-13$ & 20.9 \\
\hline
\end{tabular}

TABLE 3. $f_{3}(x)=(\sqrt{2}+\log (1+\sqrt{2}))^{-1} \sqrt{1+x^{2}}$

\begin{tabular}{rlrlr}
\hline $\mathrm{N}$ & max err1 & ratio1 & max err2 & ratio2 \\
\hline 4 & $3.62 \mathrm{E}-3$ & & $1.50 \mathrm{E}-2$ & \\
9 & $2.75 \mathrm{E}-5$ & 131.6 & $1.49 \mathrm{E}-3$ & 10.1 \\
16 & $2.44 \mathrm{E}-7$ & 112.7 & $1.29 \mathrm{E}-4$ & 11.5 \\
25 & $8.33 \mathrm{E}-9$ & 29.3 & $1.48 \mathrm{E}-5$ & 8.8 \\
36 & $2.18 \mathrm{E}-10$ & 38.2 & $1.66 \mathrm{E}-6$ & 8.9 \\
49 & $6.81 \mathrm{E}-12$ & 32.0 & $1.85 \mathrm{E}-7$ & 9.0 \\
64 & $2.05 \mathrm{E}-13$ & 33.2 & $2.05 \mathrm{E}-8$ & 9.0 \\
81 & $7.16 \mathrm{E}-15$ & 28.6 & $2.26 \mathrm{E}-9$ & 9.1 \\
100 & $2.54 \mathrm{E}-16$ & 28.2 & $2.48 \mathrm{E}-10$ & 9.1 \\
\hline
\end{tabular}

TABLE 4. $f_{4}(x)=\frac{2 x}{\pi}\left(\sqrt{1-x^{4}}\right)^{-1}$

\begin{tabular}{rlrll}
\hline $\mathrm{N}$ & max err1 & ratio1 & max err2 & ratio2 \\
\hline 4 & $9.60 \mathrm{E}-3$ & & $3.53 \mathrm{E}-2$ & \\
9 & $9.39 \mathrm{E}-4$ & 10.2 & $7.08 \mathrm{E}-3$ & 5.0 \\
16 & $9.48 \mathrm{E}-5$ & 9.9 & $1.49 \mathrm{E}-3$ & 4.7 \\
25 & $7.66 \mathrm{E}-6$ & 12.4 & $3.14 \mathrm{E}-4$ & 4.8 \\
36 & $8.51 \mathrm{E}-7$ & 9.0 & $6.63 \mathrm{E}-5$ & 4.7 \\
49 & $7.86 \mathrm{E}-8$ & 10.8 & $1.39 \mathrm{E}-6$ & 4.8 \\
64 & $8.84 \mathrm{E}-9$ & 8.9 & $2.92 \mathrm{E}-6$ & 4.8 \\
81 & $8.62 \mathrm{E}-10$ & 10.3 & $6.10 \mathrm{E}-7$ & 4.8 \\
100 & $9.68 \mathrm{E}-11$ & 8.9 & $1.28 \mathrm{E}-7$ & 4.8 \\
\hline
\end{tabular}




\section{Appendix. Proof of Lemma 1 (Ganelius)}

We shall give the proof due to Ganelius. Ganelius's exposition of the proof is very concise, as the lemma is not central to his paper. Also, in the original version several of the $a_{k}$ are equal to 1 . For our purposes we must have the $a_{k}$ distinct and all unequal to 1 ; otherwise some of the $b_{k}$ used for the indefinite quadrature formula would be multiple, which would introduce derivatives of the integrand into the formula. Making the $a_{k}$ distinct and unequal to 1 involves changes at the beginning and at the end of the proof. There is also a small error in Ganelius's exposition, which we correct.

We begin by rewriting the inequality in Lemma 1 as

$$
\sum_{k=1}^{N} \log \left|\frac{x+a_{k}}{x-a_{k}}\right| \geq \pi \sqrt{N r}+r \log x-\mathcal{C}
$$

and observing that the sum may be written as

$$
\int_{0}^{1} \log \left|\frac{x+y}{x-y}\right| d \nu(y)
$$

where $d \nu$ is a discrete measure with unit masses at the points $a_{k}$. In order to determine $a_{k}$ that will give us (1), we introduce the continuous measure

$$
d \widetilde{\mu}(y)= \begin{cases}0, & \text { if } 0 \leq y \leq 1 / \varphi\left(N_{o}\right) \\ \frac{2}{\pi^{2}} \log \left(y \varphi\left(N_{o}\right)\right)^{r} y^{-1} d y & \text { if } 1 / \varphi\left(N_{o}\right)<y<1\end{cases}
$$

We shall show that

$$
\int_{0}^{1} \log \left|\frac{x+y}{x-y}\right| d \widetilde{\mu}(y) \geq \pi \sqrt{N_{o} r}+r \log x-\frac{\pi x}{2} \sqrt{N_{o} r}-\mathcal{C} .
$$

We then introduce the discrete measure $d \mu(y)$ that has unit masses at the $N_{o}+1$ points

$$
y_{k}=\varphi(k-1) / \varphi\left(N_{o}\right), \text { for } k=1,2, \ldots, N_{o}, \quad \text { and } \quad y_{N_{o}+1}=\varphi\left(N_{o}-1 / 2\right) / \varphi\left(N_{o}\right),
$$

and show that

$$
\int_{0}^{1} \log \left|\frac{x+y}{x-y}\right| d \mu(y)-\int_{0}^{1} \log \left|\frac{x+y}{x-y}\right| d \widetilde{\mu}(y) \geq-\mathcal{C},
$$

where the generic constant $\mathcal{C}$ in (2) and (3) is independent of $x$ in $[0,1]$ and of $N$. Finally, we shall use the additional points $y_{k}$, for $k=N_{o}+2, N_{o}+3, \ldots, N$, to remove the $\frac{\pi x}{2} \sqrt{N_{o} r}$ term from (2). This will establish (1), with the $a_{k}$ being the $y_{k}$.

Proof of (2). We first note that in proving (2) we need not consider $x \leq 1 / \varphi\left(N_{o}\right)$ because then the left hand side of (2) is positive while the right hand side is negative. We have

$$
\int_{0}^{1} \log \left|\frac{x+y}{x-y}\right| d \widetilde{\mu}(y)=\frac{2}{\pi^{2}} \int_{1 / \varphi\left(N_{o}\right)}^{1} \log \left|\frac{x+y}{x-y}\right| \log \left(y \varphi\left(N_{o}\right)\right)^{r} y^{-1} d y .
$$


By setting $\xi=x \varphi\left(N_{o}\right)$ and $u=y / x$ the last expression takes the form

$$
\begin{aligned}
\frac{2 r}{\pi^{2}} \int_{\frac{1}{\xi}}^{\frac{1}{x}} \log \left|\frac{1+u}{1-u}\right| \log \xi \cdot \frac{1}{u} d u+\frac{2 r}{\pi^{2}} \int_{\frac{1}{\xi}}^{\frac{1}{x}} \log \left|\frac{1+u}{1-u}\right| \log u \cdot \frac{1}{u} d u \\
=\frac{2 r}{\pi^{2}} \int_{\frac{1}{\xi}}^{\frac{1}{x}} \log \left|\frac{1+u}{1-u}\right| \log u \cdot \frac{1}{u} d u+\frac{2 r}{\pi^{2}} \int_{0}^{\infty} \log \left|\frac{1+u}{1-u}\right| \log \xi \cdot \frac{1}{u} d u \\
\quad-\frac{2 r}{\pi^{2}} \int_{0}^{\frac{1}{\xi}} \log \left|\frac{1+u}{1-u}\right| \log \xi \cdot \frac{1}{u} d u-\frac{2 r}{\pi^{2}} \int_{\frac{1}{x}}^{\infty} \log \left|\frac{1+u}{1-u}\right| \log \xi \cdot \frac{1}{u} d u
\end{aligned}
$$

The first term of (4) is bounded below because $1 / \xi \leq 1$ so that the term is bounded below by

$$
\frac{2 r}{\pi^{2}} \int_{0}^{1} \log \left|\frac{1+u}{1-u}\right| \log u \cdot \frac{1}{u} d u,
$$

which is finite. The second term of (4) is $\log \xi^{r}$ that is $\pi \sqrt{N_{o} r}+r \log x$, and for $\xi \geq 1$, i.e., for $x \geq 1 / \varphi\left(N_{o}\right)$ the integral in the third term of (4) is an increasing function of $u$ on $(0,1)$ so that the third term is bounded by

$$
\frac{2 r}{\pi^{2} \xi} \int_{0}^{1} \log \left|\frac{1+u}{1-u}\right| \log \xi \cdot \frac{1}{u}
$$

Finally, by letting $u=1 / t$

$$
\int_{0}^{x} \log \left|\frac{1+t}{1-t}\right| \frac{1}{t} d t \leq \frac{\pi^{2} x}{4},
$$

so that the last term of (4) becomes

$$
\frac{2 r}{\pi^{2}} \int_{0}^{x} \log \left|\frac{1+t}{1-t}\right| \log \xi \cdot \frac{1}{t} d t \leq \frac{\pi x}{2} \sqrt{N_{o} r}
$$

which completes the proof of $(2)$.

Proof of (3). Letting

$$
g(x, y)=\log \left|\frac{x+y}{x-y}\right|
$$

we write the left hand side of (3) as

$$
\begin{aligned}
& \frac{1}{2} g(\xi, \varphi(0))+\sum_{k=0}^{N_{o}-2}\left[\frac{1}{2} g(\xi, \varphi(k))+\frac{1}{2} g(\xi, \varphi(k+1))-\int_{k}^{k+1} g(\xi, \varphi(u)) d u\right] \\
& \quad+\frac{1}{2} g\left(\xi, \varphi\left(N_{o}-1\right)\right)+\left[g\left(\xi, \varphi\left(N_{o}-1 / 2\right)\right)-\int_{N_{o}-1}^{N_{o}} g(\xi, \varphi(u)) d u\right]
\end{aligned}
$$

where $\xi=x \varphi\left(N_{o}\right)$ and $u=y / x$ as before. The first and third terms are nonnegative and so may be ignored. Each term in the sum is the error of a trapezoidal approximation to an integral, which is nonnegative if the integrand is convex on the closed integration interval. Let $k_{o}$ be the particular $k$ for which $\xi$ lies in the interval $\left(\varphi\left(k_{o}\right), \varphi\left(k_{o}+1\right)\right)$, Ganelius states that for $k \neq k_{o}, g(\xi, \varphi(u))$ is convex on 
$[k, k+1]$. But that is not so for $k=0$. So (5) consists entirely of positive quantities, plus the quantity

$$
\begin{aligned}
& {\left[\frac{1}{2} g(\xi, \varphi(0))+\frac{1}{2} g(\xi, \varphi(1))-\int_{0}^{1} g(\xi, \varphi(u)) d u\right]} \\
& \quad+\left[\frac{1}{2} g\left(\xi, \varphi\left(k_{o}\right)\right)+\frac{1}{2} g\left(\xi, \varphi\left(k_{o}+1\right)\right)-\int_{k_{o}}^{k_{o}+1} g(\xi, \varphi(u)) d u\right] \\
& \quad+\left[g\left(\xi, \varphi\left(N_{o}-1 / 2\right)\right)-\int_{N_{o}-1}^{N_{o}} g(\xi, \varphi(u)) d u\right]
\end{aligned}
$$

which we must prove is bounded from below.

The boundedness of the first term in (6) follows from its continuity as a function of $\xi$ and the fact that it approaches 0 as $\xi$ approaches infinity. The same reasoning would apply to the last two terms if $N_{o}$ and $k_{o}$ were bounded; thus we are concerned only with large (approaching infinity) $N_{o}$ and $k_{o}$ in the last two terms in (6).

For large $k_{o}$, the second term in (6) is bounded because

$$
\frac{1}{2} g\left(\xi, \varphi\left(k_{o}\right)\right), \frac{1}{2} g\left(\xi, \varphi\left(k_{o}+1\right)\right) \geq \frac{1}{4} \log k_{o}+O(1),
$$

and

$$
\int_{k_{o}}^{k_{o}+1} g(\xi, \varphi(u)) d u \leq \frac{1}{2} \log k_{o}+O(1) .
$$

For $\xi \leq \varphi\left(N_{o}-2\right)$, there is no singularity in the integral of the last term so that the last term is equal to

$$
g\left(\xi, \varphi\left(N_{o}-1 / 2\right)\right)-g(\xi, \beta)
$$

for some $\beta$ in $\left[\varphi\left(N_{o}-1\right), \varphi\left(N_{o}\right)\right]$. For any fixed $\xi$,

$$
g\left(\xi, \varphi\left(N_{o}\right)\right) \leq g(\xi, x) \leq g\left(\xi, \varphi\left(N_{o}-1\right)\right)
$$

for all $x$ in that interval, so (7) is bounded in absolute value by

$$
g\left(\xi, \varphi\left(N_{o}-1\right)\right)-g\left(\xi, \varphi\left(N_{o}\right)\right)
$$

which is less than equal to

$$
g\left(\varphi\left(N_{o}-2\right), \varphi\left(N_{o}-1\right)\right)-g\left(\varphi\left(N_{o}-2\right), \varphi\left(N_{o}\right)\right) .
$$

As $N_{o}$ grows, each of the last two terms above is equal to

$$
\frac{1}{2} \log N_{o}+O(1)
$$

so that the last term of (6) is bounded below for $\xi \leq \varphi\left(N_{o}-2\right)$.

For $\varphi\left(N_{o}-2\right)<\xi<\varphi\left(N_{o}\right)$, it follows that

$$
g\left(\xi, \varphi\left(N_{o}-1 / 2\right)\right) \geq \frac{1}{2} \log N_{o}+O(1)
$$

and

$$
\int_{N_{o}-1}^{N_{o}} g(\xi, \varphi(u)) d u \leq \frac{1}{2} \log N_{o}+O(1)
$$

so that the last term in (6) is bounded below. Hence (3) is established. 
Our last step in proving Lemma 1 is the elimination of the $(\pi x / 2) \sqrt{N_{o} r}$ term in (2). For that we use the fact that

$$
\log \left|\frac{x+y}{x-y}\right| \geq 2 x \text { for } 0 \leq x \leq 1
$$

as long as

$$
y \geq \frac{e^{2}-1}{e^{2}+1}=0.76 \cdots,
$$

because each of the $y_{k}$ is greater than equal to 0.8 for $k \geq N_{o}+2$. So we have

$$
\sum_{k=N_{o}+2}^{N} g\left(x, y_{k}\right) \geq \frac{\pi x}{2} \sqrt{N_{o} r} \text {. }
$$

The fact that

$$
\pi \sqrt{N_{o} r} \geq \pi \sqrt{N r}-\mathcal{C}
$$

where $\mathcal{C}$ dependent on $r$ but independent of $N$, completes the proof of Lemma 1 ,

with the $a_{k}$ being the $y_{k}$.

\section{REFERENCES}

[1] J. E. Andersson, Optimal quadrature of $H^{p}$ functions, Math. Z. 172 (1980), 55-62.

[2] J. E. Andersson and B. D. Bojanov, A note on the optimal quadrature in $H^{p}$, Numer. Math. 44 (1984), 301-308.

[3] B. D. Bojanov, optimal rate of integration and $\epsilon$-entropy of a certain class of analytic functions (in Russian), Mat. Zametki 14, 1(1973), 3-10. [English transl.: Math. Notes 14 (1973) 551-556.]

[4] - Best quadrature formula for a certain class of analytic functions, Zastosowania Matematyki Appl. Mat. XIV, 3 (1974), 441-447.

[5] P. L. Duren, Theory of $H^{p}$ spaces, Academic Press, San Diego (1970)

[6] T. Ganelius, Rational approximation in the complex plane and on the line, Ann. Acad. Sci. Fenn. Ser. A. I. 2 (1976), 129-145.

[7] - Some extremal functions and approximation, Fourier analysis and approximation theory, Proceedings of a Colloquium (Budapest 1976), 371-381, Amsterdam-Oxford-New York, North Holland (1978).

[8] S. Haber, The tanh rule for numerical integration, SIAM J. Numer. Anal. 14 (1977), 668-685.

[9] _ Two formulas for numerical indefinite integration, Math. Comp. 201 (1993), 279296

[10] R. B. Kearfott, A sinc approximation for the indefinite integral, Math. Comp., 41 (1983), 559-572.

[11] H. L. Loeb, and H. Werner, Optimal numerical quadrature in $H^{p}$ spaces, Math. Z. 138 (1974), 111-117.

[12] D. J. Newman, Rational approximation to $|x|$, Michigan Math. J. 11 (1964), 11-14.

[13] Q Quadrature formula for $H^{p}$ functions, Math. Z. 166 (1979), 111-115.

[14] C. Schwartz, Numerical integration of analytic function, J. Comput. Phys. 4 (1969), 19-29.

[15] K. Sikorski, Optimal Quadrature Algorithms in $H_{p}$ Spaces, Numer. Math. 39 (1982), 405-410.

[16] K. Sikorski, F. Stenger, J. Schwing, Algorithm 614, A Fortran Subroutine for Numerical Integration in $H_{p}$, ACM TOMS, v.10 (1984), 152-160.

[17] K. Sikorski, F. Stenger, Optimal Quadratures in $H_{p}$ Spaces, ACM TOMS, v.10 (1984), 140151.

[18] F. Stenger, Numerical methods based on Whittaker cardinal, or sinc functions, SIAM Rev. 23 (1981), 165-224.

Trinity College, 10505 Oakton Ridge Court, Oakton, Virginia 22124

E-mail address: aeyoung@prodigy.net

Temple University, Mosaryk 1, Jerusalem, Israel 